\title{
The Effect of Type of Communication by Physical Therapists on Patient Satisfaction and Revisit Intention
}

\author{
Eun-Ji Kim¹, Gyeong Seop Sim², Dong-Hoon Kim¹ \\ 'Department of Physical Therapy, Ansan University, Ansan, Republic of Korea; ${ }^{2}$ Department of Health Exercise Rehabilitation, Yeoju Institute of \\ Technology, Yeoju, Republic of Korea
}

Purpose: This study sought to identify the effect of various types of communication by physical therapists on patient satisfaction and revisit intention.

Methods: A total of 212 patients who received physical therapy in Seoul and the Gyeonggi province were surveyed on patient satisfaction and revisit intentions depending on the types of communication by physical therapists. Frequency and correlation analyses were conducted to process the data using the SPSS 22.0 program, and the AMOS 18.0 statistical package was used for confirmatory factor analysis. A structural equation modeling analysis was also performed to verify the model and the hypothesis that was set up in the present paper.

Results: The survey showed that patient satisfaction correlates positively and significantly with patient-centric communication, physical factors, and spatial behavior. A significant negative correlation was also found between patient satisfaction and therapist-centered communication. Patient satisfaction positively correlated with revisit intentions.

Conclusion: These results showed that there was a positive correlation between the communication of the physical therapist and the satisfaction recognized by a patient. This implies that the patient satisfaction with a physical therapist increases as the communication skills of a physical therapist improve. These findings suggest that a physical therapist must adopt patient-centric communication styles, physical factors, and spatial behavior. This study was important as it generated basic data for the formation of a therapeutic relationship through efficient and promotional communication skills. It recognizes the importance of communication between a physical therapist and a patient as the indispensable factor for interaction.

Keywords: Communication, Non-verbal communication, Patient satisfaction

\section{서 론}

최근 글로벌 환경의 변화, 소득의 증가, 노령화 현상의 심화로 의료서 비스에 대한 수요가 증가하고 있다. 이러한 의료 서비스에 대한 수요 증가로 국내 의료시장은 급격한 양적 성장을 이루었으나, 경쟁의 심 화로 인하여 중소 병원의 경영난은 악화되고 있다. ${ }^{2}$ 이러한 경쟁에서 우위를 확보하고자, 공급자 중심의 시장에서 소비자가 주도하는 고 객중심경영을 받아들이고 있다. ${ }^{3}$

의료기관의 질적 차별화를 위해 행정 서비스, 대기 편의, 의료 시설 및 환경의 개선 등의 물리적 환경의 투자와 더불어 인적 서비스 수준 의 향상이 중요한 요소로 부각되고 있다. ${ }^{4-5}$ 의료서비스 이용자 들은 의료진에 대한 신뢰를 중요하게 생각하며, 이러한 신뢰는 진료 및 치
료행위를 하는 의사의 유형적 행동을 통해 이루어지게 된다. ${ }^{6}$ 의료진 료 행위의 가장 기본이 되는 것은 커뮤니케이션으로, 의사와 환자 관 계의 신뢰는 이러한 커뮤니케이션으로 시작된다고 볼 수 있다.7-9

커뮤니케이션은 언어적 커뮤니케이션과 비언어적 커뮤니케이션으 로 분류할 수 있다. 언어적 커뮤니케이션은 커뮤니케이션의 가장 큰 부분을 차지하며, 단어, 말투, 억양 등으로 의미를 전달하는 것을 말 한다. ${ }^{10}$ 선행연구에서 언어적 커뮤니케이션은 의사-환자 간의 긍정적 인 관계를 유지할 수 있는 '환자 중심형, 협력형'과, 권위적이고 치료내 용에 관한 대화 외에 사적인 질문은 거의 하지 않는 '의사 중심형, 통 제형'으로 분류하고 있다. ${ }^{11-12}$

비언어적 커뮤니케이션은 표정, 몸짓 등 언어를 제외한 형태로 의 도행동과 비 의도행동을 포함한 모든 것을 의미한다..$^{13} \mathrm{Lee}{ }^{14}$ 는 호텔을 
대상으로 비언어적 커뮤니케이션 요소가 고객 만족에 유의한 영향 을 미친다고 하였으며, $\mathrm{Kim}$ 과 $\mathrm{Seo}^{15}$ 는 의료서비스 제공자의 비언어적 커뮤니케이션이 고객만족과 고객행동의도에 유의한 영향을 미친다 고하였다.

물리치료사는 의사의 지도하에 진료 또는 의화학적 검사에 종사 하는 의료기사로 물리적인자치료, 기능훈련, 교정운동 및 재활훈련 등의 업무에 종사하는 자로 규정되어 있다. ${ }^{16}$ 물리치료사는 환자들을 대상으로 다양한 직접적인 의료서비스를 제공하고 있어 환자와의 의 사소통이 굉장히 중요하다. 또한 물리치료 적용 시간은 평균 30-60분 으로 의사보다 의료서비스 제공 시 환자와 함께하는 시간이 길기 때 문에 언어적, 비언어적 커뮤니케이션 상황에 처하는 시간이 길어 환 자들의 의료서비스 만족도에 많은 영향을 미친다. 그러므로 물리치 료사가 환자에게 신뢰를 얻고 만족감을 주기 위해서는 대인관계능력 과 더불어 적절한 의사소통 능력이 필요하다. ${ }^{17}$ 그럼에도 불구하고, 선행연구에서는 물리치료실에 내원하는 환자의 일반적인 특성, 병원 접근도, 의료기관의 형태, 도시규모 등의 요인에 따른 만족도 조사연 구에만 국한되어 있었다. ${ }^{18}$ 선행연구 만으로는 물리치료사의 커뮤니 케이션이 환자 만족도에 어떤 영향을 미치는지 알 수 없었다. 따라서 환자가 느끼는 의료 서비스에 대한 만족과 재방문 의사를 좌우하는 물리치료사의 커뮤니케이션 유형을 규명할 필요가 있다. 본 연구는 '물리치료사의 커뮤니케이션 유형에 따라 환자만족과 재방문의사에 미치는 영향이 차이가 있을 것이다라는 가설을 세우고, 변수 간의 영
향을 분석하여 물리치료사의 커뮤니케이션 중요성을 언급하고자 하 였다.

\section{연구 방법}

\section{1. 연구대상}

본 연구는 서울, 경기 소재의 종합병원, 병원 및 의원 등에서 물리치 료를 받은 환자들 대상으로, 설문 참여에 동의한 218 명을 대상으로 Park과 $\mathrm{Kim}^{19}$ 의 선행연구를 토대로 본 연구 목적에 맞게 수정 보완한 후 커뮤니케이션 유형과 환자만족도, 재방문의사 척도를 설문조사 하였다. 모든 문항은 5점 리커트 척도로 '전혀 그렇지 않다', '그렇지 않 다, '보통이다', '그렇다', '매우 그렇다' 5 점을 부여해 사용하였다(Table 1). 수집기간은 2021년 6월 5일부터 19일까지, 온라인을 통하여 2주간 실시하였으며 총 218 부 중 설문 응답이 완료되지 않은 6 부를 제외하 고, 212 부의 설문을 분석하였다.

\section{2. 실험방법}

1) 연구모형

본 연구의 모형은 물리치료사의 언어적 커뮤니케이션 유형, 비언어적 커뮤니케이션 유형, 환자 만족, 재방문 의사의 4 개의 범주로 구성하였 다. 본 연구는 물리치료사의 커뮤니케이션 유형에 따른 환자 만족과 재방문 의사에 미치는 영향 분석을 확인하고자 하였다(Figure 1).

Table 1. Reliability of the questionnaire

\begin{tabular}{|c|c|c|}
\hline Category & Questions & Cronbach's $\alpha$ \\
\hline \multirow[t]{4}{*}{ Patient-centered } & The physical therapist serves me well & 0.778 \\
\hline & The physical therapist listens carefully to my minor symptoms and opinions & \\
\hline & The physical therapist is sincerely trying to relieve my discomfort (pain) & \\
\hline & The physical therapist creates a very comfortable atmosphere for conversation & \\
\hline \multirow[t]{4}{*}{ Therapist-centered } & The physical therapist serves me with a bit of a stiff and authoritative attitude & 0.912 \\
\hline & The physical therapist uses extreme language in conversations with me & \\
\hline & The physical therapist is strict & \\
\hline & The physical therapist doesn't attempt anything other than therapy-related conversations & \\
\hline \multirow[t]{5}{*}{ Body language } & The physical therapist greets me with a smile when I enter the treatment room & 0.922 \\
\hline & The physical therapist explains the treatment with proper eye contact & \\
\hline & The physical therapist listens to me and nods & \\
\hline & The physical therapist listens to my symptoms well & \\
\hline & The physical therapist uses gestures appropriately to easily explain about treatment & \\
\hline \multirow[t]{3}{*}{ Spatial behavior } & The physical therapist's desk is neat and clean & 0.860 \\
\hline & The physical therapy room is neat and clean & \\
\hline & The physical therapy room is structured for the convenience of the patient & \\
\hline \multirow[t]{4}{*}{ Patient satisfaction } & I am satisfied with the treatment of the physical therapist & 0.965 \\
\hline & I am satisfied with the communication style of the physical therapist & \\
\hline & I am satisfied with the attitude of the physical therapist who treated me & \\
\hline & I am satisfied with the overall things of the physical therapist who treated me & \\
\hline \multirow[t]{2}{*}{ Revisit intention } & I would like to visit this therapist again for treatment & 0.845 \\
\hline & I would like to receive the treatment by first selecting the physical therapist who treated me & \\
\hline
\end{tabular}




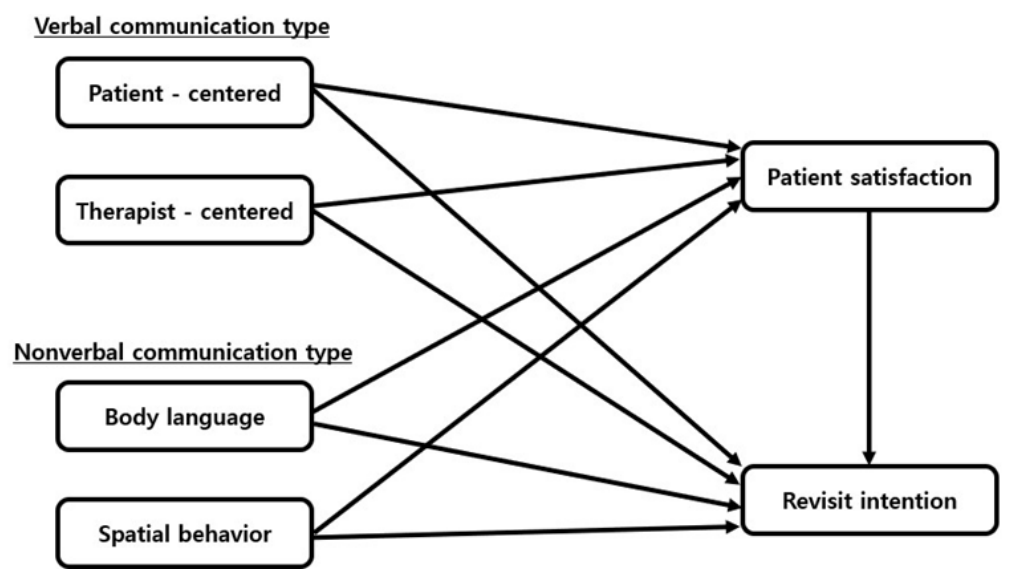

Figure 1. Study model.

\section{2) 물리치료사의 커뮤니케이션 유형}

커뮤니케이션 유형은 언어적 커뮤니케이션 유형과 비언어적 커뮤니 케이션 유형으로 분류하였다. 언어적 커뮤니케이션 유형은 Park과 $\mathrm{Kim}^{19}$ 이 선행연구를 토대로 제시한 문항을, '치료사중심형' 4 문항, ‘환 자 중심형' 4 문항으로 본 연구 목적에 맞게 총 8 문항으로 수정 보완하 였다. '치료사중심형' 문항의 신뢰도는 Cronbach's $\alpha=0.778$, '환자중심 형' 문항의 신뢰도는 Cronbach's $\alpha=0.912$ 였다.

비언어적 커뮤니케이션 유형은 Park과 $\mathrm{Kim}^{19}$ 이 선행연구를 토대로 제시한 문항을 '신체적 언어' 5 문항, '공간적 행위' 3 문항으로 본 연구의 목적에 맞게 총 8 문항으로 수정 보완하였다. 신체적 언어 문항의 신뢰 도는 Cronbach's $\alpha=0.922$, 공간적 행위 문항의 신뢰도는 Cronbach's $\alpha$ $=0.860$ 였다(Table 1 ).

\section{3) 환자 만족도}

환자 만족도는 Park과 $\mathrm{Kim}^{19}$ 이 선행연구를 토대로 제시한 문항을 '치 료사에 대한 만족' '커뮤니케이션의 유형에 대한 만족' '치료사의 태 도 만족' '전반적인 만족' 분류하여 각 1 문항 총 4 문항으로 수정 보완 하였다. 문항의 신뢰도는 Cronbach's $\alpha=0.965$ 였다(Table 1).

\section{4) 재방문 의사}

재방문 의사는 Park과 $\mathrm{Kim}^{19}$ 이 선행연구를 토대로 제시한 문항을 본 연구의 목적에 맞게 총 2 문항으로 수정 보완하였다. 문항의 신뢰도는 Cronbach's $\alpha=0.845$ 였다(Table 1 ).

\section{3. 분석방법}

통계분석을 위하여 SPSS Ver 22.0와 AMOS Ver 18.0을 이용하였다. 측 정변수의 신뢰성 검증을 위해 Cronbach's $\alpha$ 계수를 이용하였다. 연구 대상자의 인구통계적 특성은 빈도분석을 실시하였고, 변수 간의 상 관관계를 알아보기 위하여 SPSS Ver 22.0를 이용해 Pearson 상관계수
Table 2. Demographic characteristics of study subjects

\begin{tabular}{|c|c|c|c|}
\hline & Classification & $\begin{array}{c}\text { Frequency } \\
\text { (persons) }\end{array}$ & $\begin{array}{c}\text { Percentage } \\
(\%)\end{array}$ \\
\hline \multirow[t]{2}{*}{ Gender } & Male & 107 & 50.5 \\
\hline & Female & 106 & 49.5 \\
\hline \multirow[t]{5}{*}{ Age } & 20's & 71 & 33.5 \\
\hline & 30 's & 54 & 25.5 \\
\hline & 40 's & 46 & 21.7 \\
\hline & 50's & 35 & 16.5 \\
\hline & over 60's & 6 & 2.8 \\
\hline \multirow[t]{7}{*}{ Job } & Public officials & 4 & 1.9 \\
\hline & Professions & 28 & 13.2 \\
\hline & Self-employed & 16 & 7.5 \\
\hline & Housewives & 13 & 6.1 \\
\hline & Students & 56 & 26.4 \\
\hline & Office workers & 73 & 34.4 \\
\hline & Etc. & 22 & 10.4 \\
\hline \multirow[t]{2}{*}{ Marriage } & Married & 100 & 47.2 \\
\hline & Unmarried & 112 & 52.8 \\
\hline \multirow{5}{*}{$\begin{array}{l}\text { Last educational } \\
\text { background }\end{array}$} & Below high school graduate & 49 & 52.8 \\
\hline & College graduate & 63 & 29.7 \\
\hline & University graduate & 80 & 37.7 \\
\hline & In graduate school & 10 & 4.7 \\
\hline & Graduate school graduation & 10 & 4.7 \\
\hline \multirow[t]{5}{*}{ Monthly income } & No income & 49 & 23.1 \\
\hline & Less than 2 million won & 38 & 17.9 \\
\hline & 2 million won to 3 million won & 61 & 28.8 \\
\hline & 3 million won to 4 million won & 38 & 17.9 \\
\hline & Over 4 million won & 26 & 12.3 \\
\hline
\end{tabular}

를 구하였다. 설문문항의 타당성을 확인하기 위하여 확인적 요인분 석을 실시하였고, 가설검증을 위해 AMOS Ver 18.0으로 공분산분석 을 실시하였다.

결 과

\section{1. 연구 대상자의 인구통계적 특성}

연구 대상자의 인구통계적 특성은 다음과 같다. 성별은 ‘남성’이 107 명(50.5\%), ‘여성'이 105명(49.5\%)으로 나타났고, 연령은 ‘20대’는 71명 
(33.5\%), ‘30대’는 54명(25.5\%), '40대’는 46명(21.7\%), '50대'는 35명(16.5\%), '60대 이상'은 6명(2.8\%)이었다(Table 2).

직업은 ‘공무원’이 4명(1.9\%), '전문직’이 28명(13.2\%), ‘자영업’이 16명 (7.5\%), '주부’가 13명(6.1\%), ‘학생’이 56명(26.4\%), ‘회사원'이 73명(34.4\%), '기타'가 22명(10.4\%)이었고, 결혼은 ‘기혼’이 100명(47.2\%), ‘미혼’이 112 명(52.8\%)이었다(Table 2).

최종 학력은 '고졸 이하'가 49명(23.1\%), '전문대졸'이 63명(29.7\%), '대 졸'이 80명(37.7\%), '대학원 재학'이 10명(4.7\%), '대학원 졸업'이 10 명 (4.7\%)이었고, 월소득은 ‘수입 없음’이 49명(23.1\%), '200만 원 미만’이 38 명(17.9\%), '200-300만 원 미만’이 61명(28.8\%), ‘300-400만 원 미만’이 38 명(17.9\%), '400만 원 이상'이 26명(12.3\%)이었다(Table 2).

\section{2. 조사도구의 타당성}

본 연구의 조사도구에 대한 타당도 평가를 위해 확인적 요인분석을 실시하였다. 측정모형의 타당도 평가 결과는 $\chi^{2} / \mathrm{df}=1.53, \mathrm{CFI}=0.97$, $\mathrm{TLI}=0.96, \mathrm{RMSEA}=0.05$ 로 나타났다(Table 3 ). 일반적으로 $\chi^{2} / \mathrm{df}<3$ 이 면 모형이 양호한 것으로 판단하고, CFI (comparative fit index) 3 와 TLI (Turker-Lewis index)는 0.9 이상이면 모형이 우수한 것으로 판단하며, RMSEA (root mean square error of approximation) 값은 0.10 미만이면 받아들일 수 있는 수준의 모형으로 판단하는데, 본 연구의 조사도구 는 기준치를 모두 충족하므로, 요인분석 적합도는 양호한 것으로 판
단되었다. 또한 모든 문항에 대한 요인 부하량이 통계적으로 유의하 므로 개념타당도를 확보하였다고 할 수 있다 $(\mathrm{p}<0.05)$.

\section{3. 요인 간 상관관계}

\section{1) 환자 만족}

환자 중심형 커뮤니케이션 $(\mathrm{r}=0.87)$ 과 신체적 언어 $(\mathrm{r}=0.86)$, 공간적 행 위 $(\mathrm{r}=0.76)$ 요소는 환자 만족도와 유의미한 양 $(+)$ 의 상관관계가 있는 것으로 나타났다. 반면 치료사 중심형 커뮤니케이션은 환자 만족 $(\mathrm{r}=$ $-0.38)$ 과 유의미한 음(-)의 상관 관계가 있는 것으로 나타났다 $(\mathrm{p}<0.05)$ (Table 4, Figure 2).

\section{2) 재방문 의사}

환자 중심형 커뮤니케이션 $(\mathrm{r}=0.75)$ 과 신체적 언어 $(\mathrm{r}=0.75)$, 공간적 행 위 $(\mathrm{r}=0.72)$ 요소는 재방문 의사와 유의미한 양 $(+)$ 의 상관관계가 있는 것으로 나타났다. 반면 치료사 중심형 커뮤니케이션은 재방문 의사 $(\mathrm{r}=-0.33)$ 와 유의미한 음(-)의 상관 관계가 있는 것으로 나타났다 $(\mathrm{p}<$ 0.05) (Table 4, Figure 2).

\section{4. 모형 검증}

\section{1) 모형 적합도}

물리치료사의 언어적 커뮤니케이션 유형, 비언어적 커뮤니케이션 유

Table 3. Confirmatory factor analysis

\begin{tabular}{|c|c|c|c|c|c|}
\hline Path & B & S.E & $\beta$ & C.R & $\mathrm{p}$ \\
\hline Patient-centered $\rightarrow$ Patient-centered 1 & 1 & & & & \\
\hline Patient-centered $\rightarrow$ Patient-centered 2 & 1.10 & 0.06 & 0.87 & 16.80 & $<0.001^{* * *}$ \\
\hline Patient-centered $\rightarrow$ Patient-centered 3 & 1.19 & 0.06 & 0.89 & 17.33 & $<0.001^{* * *}$ \\
\hline Patient-centered $\rightarrow$ patient-centered 4 & 1.12 & 0.07 & 0.82 & 14.93 & $<0.001^{* * *}$ \\
\hline Therapist-centered $\rightarrow$ Therapist-centered 1 & 1 & & & & \\
\hline Therapist-centered $\rightarrow$ Therapist-centered 2 & 0.90 & 0.08 & 0.78 & 11.09 & $<0.001^{* * *}$ \\
\hline Therapist-centered $\rightarrow$ Therapist-centered 3 & 0.91 & 0.08 & 0.76 & 10.84 & $<0.001^{* * *}$ \\
\hline Therapist-centered $\rightarrow$ Therapist-centered 4 & 0.57 & 0.09 & 0.43 & 5.96 & $<0.001^{* * *}$ \\
\hline Body language $\rightarrow$ Body language 1 & 1 & & & & \\
\hline Body language $\rightarrow$ Body language 2 & 1.09 & 0.08 & 0.84 & 13.51 & $<0.001^{* * *}$ \\
\hline Body language $\rightarrow$ Body language 3 & 0.98 & 0.07 & 0.85 & 13.72 & $<0.001^{* * *}$ \\
\hline Body language $\rightarrow$ Body language 4 & 0.99 & 0.06 & 0.88 & 14.45 & $<0.001^{* * *}$ \\
\hline Body language $\rightarrow$ Body language 5 & 1.08 & 0.08 & 0.84 & 13.61 & $<0.001^{* * *}$ \\
\hline Spatial behavior $\rightarrow$ Spatial behavior 1 & 1 & & & & \\
\hline Spatial behavior $\rightarrow$ Spatial behavior 2 & 0.97 & 0.07 & 0.87 & 14.00 & $<0.001^{* * *}$ \\
\hline Spatial behavior $\rightarrow$ Spatial behavior 3 & 1.12 & 0.09 & 0.82 & 13.01 & $<0.001^{* * *}$ \\
\hline Patient satisfaction $\rightarrow$ Patient satisfaction 1 & 1 & & & & \\
\hline Patient satisfaction $\rightarrow$ Patient satisfaction 2 & 1.12 & 0.04 & 0.92 & 23.84 & $<0.001^{* * *}$ \\
\hline Patient satisfaction $\rightarrow$ Patient satisfaction 3 & 1.08 & 0.04 & 0.95 & 26.40 & $<0.001^{* * *}$ \\
\hline Patient satisfaction $\rightarrow$ Patient satisfaction 4 & 1.03 & 0.04 & 0.93 & 24.92 & $<0.001^{* * *}$ \\
\hline Revisit intention $\rightarrow$ Revisit intention 1 & 1 & & & & \\
\hline Revisit intention $\rightarrow$ Revisit intention 2 & 0.94 & 0.06 & 0.78 & 15.15 & $<0.001^{* * *}$ \\
\hline
\end{tabular}

$\chi^{2}=298.00, d f=194, \chi^{2} / d f=1.53, C F I=0.97, T L I=0.96$, RMSEA $=0.05$.

$C . R=$ Construct reliability, $S . E=$ Standard errors, $B=$ Unstandardized coefficient, $\beta=$ Standardized coefficient.

${ }^{*} p<0.05,{ }^{* * *} p<0.001$. 
Table 4. Patient satisfaction and revisiting intentions according to communication types

\begin{tabular}{|c|c|c|c|c|c|c|}
\hline & \multicolumn{2}{|c|}{ Verbal communication type } & \multicolumn{2}{|c|}{ Nonverbal communication type } & \multirow{2}{*}{ Patient satisfaction } & \multirow{2}{*}{ Revisit intentior } \\
\hline & Patient-centered & Therapist-centered & Body language & Spatial behavior & & \\
\hline Patient-centered & 1 & & & & & \\
\hline Therapist-centered & $-0.38^{* *}$ & 1 & & & & \\
\hline Body language & $0.85^{\star *}$ & $-0.42^{* *}$ & 1 & & & \\
\hline Spatial behavior & $0.70^{* *}$ & $-0.33^{* *}$ & $0.73^{* *}$ & 1 & & \\
\hline Patient satisfaction & $0.87^{* *}$ & $-0.38^{* *}$ & $0.86^{\star *}$ & $0.76^{* *}$ & 1 & \\
\hline Revisit intention & $0.75^{\star *}$ & $-0.33^{* *}$ & $0.75^{* *}$ & $0.72^{* *}$ & $0.82^{* *}$ & 1 \\
\hline
\end{tabular}

$r=$ correlation coefficient.

${ }^{*} \mathrm{p}<0.05,{ }^{* *} \mathrm{p}<0.01$.

\section{Verbal communication type}

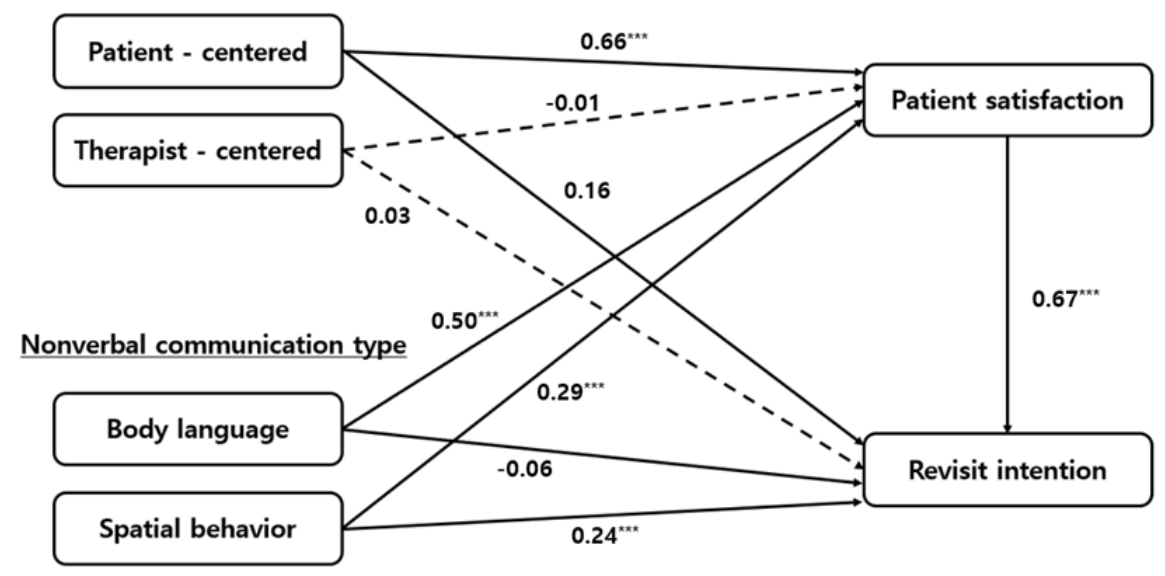

$---\rightarrow$ : Negative correlation $\longrightarrow$ : Positive correlation

Figure 2. Path coefficient of the model.

Table 5. Path coefficient of the model

\begin{tabular}{|c|c|c|c|c|c|}
\hline Path & B & S.E & $\beta$ & $C . R$ & $\mathrm{p}$ \\
\hline Patient-centered $\rightarrow$ Patient satisfaction & 0.51 & 0.04 & 0.66 & 11.77 & $<0.001^{* * *}$ \\
\hline Therapist-centered $\rightarrow$ Patient satisfaction & 0.00 & 0.03 & -0.01 & -0.30 & 0.761 \\
\hline Body language $\rightarrow$ Patient satisfaction & 0.37 & 0.04 & 0.50 & 9.30 & $<0.001^{* * *}$ \\
\hline Spatial behavior $\rightarrow$ Patient satisfaction & 0.25 & 0.04 & 0.29 & 6.19 & $<0.001^{* * *}$ \\
\hline Patient satisfaction $\rightarrow$ Revisit intention & 0.79 & 0.16 & 0.67 & 4.91 & $<0.001^{* * *}$ \\
\hline Patient-centered $\rightarrow$ Revisit intention & 0.14 & 0.09 & 0.16 & 1.50 & 0.132 \\
\hline Therapist-centered $\rightarrow$ Revisit intention & 0.02 & 0.04 & 0.03 & 0.59 & 0.552 \\
\hline Body language $\rightarrow$ Revisit intention & -0.05 & 0.07 & -0.06 & -0.71 & 0.475 \\
\hline Spatial behavior $\rightarrow$ Revisit intention & 0.24 & 0.06 & 0.24 & 3.59 & $<0.001^{* * *}$ \\
\hline
\end{tabular}

$\chi^{2}=298.00, d f=194, \chi^{2} / d f=1.53, C F I=0.97, T L I=0.96$, RMSEA $=0.05$.

$C . R=$ Construct reliability, $S . E=$ Standard errors, $B=$ Unstandardized coefficient, $\beta=$ Standardized coefficient.

${ }^{*} p<0.05,{ }^{* *} p<0.001$.

형, 환자 만족, 재방문 의사 변수 간의 관계구조를 검증하기 위해

$\mathrm{AMOS}$ 로 구조방정식 모형 분석을 실시하였다. 분석결과, $\mathrm{CFI}=0.97$, $\mathrm{TLI}=0.96$ 로 기준치인 0.9 이상으로 나타났으며, RMSEA $=0.05$ 으로 기준치인 0.1 보다 낮게 나타나 전반적으로 모형 적합도 기준을 만족 한다고 할 수 있다(Table 5).
2) 모형의 경로계수

모형의 경로계수를 살펴보면 환자 중심형에서 환자만족도로 가는 경로 $(\beta=0.66, p<0.001, C . R .=11.77)$, 신체적 언어에서 환자 만족도로 가는 경로 $(\beta=0.50, \mathrm{p}<0.001, \mathrm{C} . \mathrm{R} .=9.30)$, 공간적 행위에서 환자 만족 도로 가는 경로 $(\beta=0.29, \mathrm{p}<0.001, \mathrm{C} . \mathrm{R} .=6.19)$, 공간적 행위에서 재방 
문 의사로 가는 경로 $(\beta=0.24, p<0.001$, C.R. $=3.59)$, 환자 만족도에서 재방문 의사로 가는 경로 $(\beta=0.67, \mathrm{p}<0.001, \mathrm{C} . \mathrm{R} .=4.91)$ 가 양 $(+)$ 으로 유 의하게 나타났다. 이는 물리치료사의 커뮤니케이션 유형 중 환자 중 심형과 신체적 언어, 공간적 요소가 높을수록 환자 만족도가 높아지 며, 환자 만족이 높을수록 재방문 의사가 높아진다고 볼 수 있다(Table 5, Figure 2).

\section{고 찰}

본 연구는 물리치료사의 커뮤니케이션 유형이 환자 만족도와 재방문 의사에 미치는 영향을 분석하여 물리치료사의 커뮤니케이션 중요성 을 알아보고자 하였다.

모형의 경로계수 결과를 보면, 물리치료사의 커뮤니케이션 유형 중 언어적 커뮤니케이션 중 ‘환자 중심형' 유형이, 비언어적 커뮤니케 이션 중 '신체적 언어', '공간적 행위' 유형이 환자 만족으로 가는 경로 에 양의 상관관계를 보여 유의한 영향을 미쳤고, 언어적 커뮤니케이 션 중 '치료사 중심형' 유형이 음의 상관관계를 보여 유의하게 영향을 미치지 않는 것으로 분석되었다. 또한 환자 만족은 언어적 커뮤니케 이션의 ‘환자 중심형'과 비언어적 커뮤니케이션의 '신체적 언어' 유형 에서 만족이라는 매개변수를 거쳐야 '재방문 의사'에 유의하게 영향 을 미치는 것을 알 수 있었다. 이 결과는 환자의 만족과 병원 재방문 을 위해 물리치료사가 현장에서 보완할 수 있는 방법은 물리치료사 의 커뮤니케이션이 '치료사 중심형'에서 '환자 중심형'으로 변화되어 야 하며, 신체적 언어 사용이 증가되어야 한다는 것을 의미한다. 이는 의사의 커뮤니케이션 중 '환자 중심형', 비언어적 커뮤니케이션 중 '신 체적 언어' 유형이 환자 만족과 재방문 의사에 영향을 미친다는 Park 과 $\operatorname{Kim}^{19}$ 의 연구 결과와 유사하다. Park과 $\operatorname{Kim}^{19}$ 은 의사는 환자의 이 야기에 성의 있게 경청해야 한다고 제시하며, 대부분의 의사들이 환 자 이야기가 15 초가 넘어가면 중간에 대화를 중단하고 자신의 말만 하는 실태를 바꿔야 한다고 제안하였다. 따라서, 치료사들이 환자의 이야기를 경청하고, 치료 과정을 설명할 때 환자가 잘 이해하고 있는 지 확인하는 커뮤니케이션 과정이 필요하다고 생각된다. 의료서비스 제공자의 비언어적 커뮤니케이션이 고객만족과 고객행동의도에 미 치는 영향에 대해 연구한 $\mathrm{Kim}$ 과 $\mathrm{Yu}^{20}$ 의 연구에서도 유사한 결과가 보고되었고, 특히 고객만족에 의료서비스 제공자의 신체적 언어의 역할이 중요하며, 서비스 제공자의 신체적 언어는 고객으로 하여금 심리적으로 덜 불안하게 할 뿐만 아니라 제공받는 서비스에 대해서 도 높은 만족을 지각하게 한다고 제시했다. 그러므로, 신체적 언어 사 용을 증가하는 방법으로 치료사가 환자와 대면할 때, 환자의 이해를 도울 수 있는 손동작과 몸동작, 고개 끄덕임과 미소로 환자를 대할 것을 제안한다.
본 연구에서 비언어적 커뮤니케이션의 '공간적 행위' 유형이 '환자 만족과 '재방문 의사' 모두 유의하게 영향을 미친다는 결과는 외래 환자를 대상으로 한 간호사의 비언어적 커뮤니케이션과 만족도의 관 계를 연구한 Lee ${ }^{21}$ 의 연구결과와 다른 결과를 보였다. 또한 의사의 비 언어적 커뮤니케이션 중 '공간적 행위'가 영향을 미치지 않는다는 Park과 Kim ${ }^{19}$ 의 연구 결과와도 다른 결과를 보였다. 이는 간호사, 의 사와 환자 간 상호교류 시간이 물리치료사 보다 짧기 때문에 물리치 료사와의 대화 중 공간적 행위'에 더 많은 영향을 받았을 것으로 사 료된다. 입원환자가 지각하는 간호사의 비언어적 커뮤니케이션과 환 자 만족도를 연구한 $\mathrm{Noh}^{22}$ 의 연구 결과에서도 외래환자를 대상으로 한 이전의 연구보다 입원 환자를 대상으로 한 연구에서 더 높은 만족 도의 결과를 얻어 외래 환자보다 입원 환자와의 교류시간이 이 결과 의 차이를 보인다고 분석하였다. 서비스 제공자 주위의 지저분함, 무 질서는 고객으로 하여금 서비스 제공자의 능력과 서비스 임무를 의 심하는 원인이 되며,23 서비스 제공자와 고객의 적절한 거리조절은 고 객만족과 높은 상관관계를 가지고 있다. ${ }^{24}$ 따라서 본 연구에서 치료 사와 환자 간의 원활한 커뮤니케이션이 이루어지기 위해서는 치료실 이 청결하고 치료사의 복장이 단정하며 치료실의 공간배치가 이동 이 편리하고 장애물로 느껴지는 사물의 배치가 없어야 한다고 생각 된다.

본 연구의 의의는 물리치료사의 커뮤니케이션 형태를 분석하고 환 자의 만족도와 재방문을 위하여 현장에서 실천할 수 있는 방법을 제 시한다는 것이다. 얻어진 결론은 다음과 같다. 의료 현장에서 물리치 료사와 환자 간 더욱 큰 신뢰감을 형성하기 위해서는 '환자 중심형'의 언어적 커뮤니케이션과 '신체언어, '공간적 행위' 유형의 비언어적 커 뮤니케이션이 증가하여야 하며, 실제적 행위로는 환자와의 대화에서 환자 이야기에 경청하기, 고개 끄덕임과 미소로 대화에 응하기, 대화 시 환자와의 적당한 거리를 두기, 대화가 이루어지는 치료실 환경 개 선하기 등을 제시한다.

본 연구의 한계점은 다음과 같다. 첫째, 물리치료사와 환자에 관한 연구에서 커뮤니케이션 요소와 만족도, 재방문 의사의 관계에 대한 연구는 미비한 실정이기 때문에 연구를 통한 비교에 어려움이 있었 다. 둘째, 병원의 특수성, 물리치료실의 특수성과 환경에 대하여 모두 고려할 수 없었다. 따라서 향후 연구에서는 다양한 병원과 지역으로 확대하여 연구를 확장할 필요가 있다고 생각된다.

\section{REFERENCES}

1. Lee SY, Choi SH, Ha KJ et al. An investigation study on the role and performance ability of physical therapists in the community care system for elderly. J Kor Phys Ther. 2020;32(4):266-71.

2. Jung OK, Kim IK, Park C. The effects of service expertise on customer 
satisfaction, trust, and loyalty. Academy of Customer Satisfaction Management. 2011;13(1):57-79.

3. Lee SH, Choi KS, Kang MG et al. Measuring expectations in assessment of consumer satisfaction by SERVQUAL. Korean J Health Policy Admin. 2000;10(3):155-68.

4. Lee HJ, Song JM. Development of the korean version of ICF e-learning tool. J Kor Phys Ther. 2019;31(2):88-93.

5. Song E, Kim G. Utilization characteristics and user satisfaction by type of physical therapy service in senior centers. J Kor Phys Ther. 2018;30 (4):151-7.

6. Lee JH, Choi JM. A study on the relationship between patient's medical communication, reliance and satisfaction to dental hygienist. J Korean Soc Dent Hyg. 2012;12(5):1017-27.

7. Ha JF, Longnecker N. Doctor-patient communication: a review. Ochsner J. 2010;10(1):38-43

8. Lee DW. A conversational analysis of 'doctor-patient' communication: in search of the interpersonal communication problems and solutions. Korean J Journal Commun Stud. 2000;45(1):232-65.

9. Kim MJ. Study on the factors influencing doctor's communication styles: Individual characteristics and patient-centered trait. Korean J Journal Commun Stud. 2009;53(3):146-72.

10. Oh MY. Factor analysis on evaluating the communication competence of the panelist forum: focused on analysis of factors of nonverbal communication. Chungang University. Dissertation of Doctorate Degree. 2001.

11. Ben-Sira Z. Affective and instrumental components in the physicianpatient relationship: An additional dimension of interaction theory. JHSB. 1980;21(6):170-80.

12. Park SD. The effect of doctors communication style in clinic and outpatient gender on the nature of medical customers word of mouth. Pukyong National University. Dissertation of Master's Degree. 2012.

13. Son SM. Dose motor Inhibition response training using stop-signal paradigm influence execution and stop performance?. J Kor Phys Ther. 2020;32(2):70-4.
14. Lee JS. The effects of nonverbal communication of service encounters on guest satisfaction and service loyalty: focused on hotel. KSMS. 2008; 9(1):25-46.

15. Kim YK, Seo MS. The impact of the nonverbal communication of the medical service provider on the customer satisfaction and customer behavior. J Consumpt Cult. 2006;9(3):155-84.

16. Lee BM. A study on the plan to make physical therapists practice more efficiently in Korea. Yongin University. Dissertation of Master's Degree. 2001.

17. Jang WH, Kim SH, No SY et al. Effects of slime program on hand function and problem behavior of adults with intellectual disabilities. J Kor Phys Ther. 2020;32(4):250-7.

18. Kim TH, Jee HJ, Bae CW et al. Social distancing in COVID-19: what are the implications for musculoskeletal problems and the quality of life?. J Kor Phys Ther. 2021;33(2):84-90.

19. Park J, Kim DH. The effects of medical doctors communication styles and nonverbal communication elements on patients satisfaction and revisiting intention: Implication of CEO from hospital. Journal of CEO and Management Studies. 2016;19(2):121-38.

20. Kim YK, Yu JP. A study on verbal and non-verbal communication of medical service provider. J Korea Service Management Society. 2008;9 (1):225-58.

21. Lee YS. A study on the relationship between the nurse's communication and outpatient's trust, satisfaction. Ewha Womans University. Dissertation of Master's Degree. 2011.

22. Noh JR. Relationship of nurse's nonverbal communication to patient satisfaction at hospital. Catholic University of Pusan. Dissertation of Master's Degree. 2012.

23. Kim JH. The effect of nonverbal communication by service workers on customer satisfaction: focused on beauty-industry businesses. Daejeon University. Dissertation of Master's Degree. 2004.

24. Cho JE. Effect of non-verbal communication of airline service providers on service quality perception \& satisfaction. J Korea Academic Society of Tourism Management. 2018;33(5):221-36. 